How to reference this article

Malczewska, B. (2019). Nomi e aggettivi qualificativi riguardanti la lepre nella lingua venatoria italiana e polacca. Italica Wratislaviensia, 10(1), 133-154.

DOI: http://dx.doi.org/10.15804/IW.2019.10.1.6

Beata Malczewska

Uniwersytet Pedagogiczny im. KEN w Krakowie

beata.malczewska@up.krakow.pl, ORCID: 0000-0001-5667-6243

\title{
NOMI E AGGETTIVI QUALIFICATIVI RIGUARDANTI LA LEPRE NELLA LINGUA VENATORIA ITALIANA E POLACCA
}

\author{
NOUNS AND ADJECTIVES DESCRIBING \\ CHARACTERISTICS OF THE HARE IN POLISH \\ AND ITALIAN HUNTING LEXIS
}

\begin{abstract}
This paper expounds the results of a query conducted in terminological resources related to the hare's characteristics and features a subsequent comparison of findings. The approach is semantically-oriented and consists of qualitatively and quantitatively processed Polish and Italian data. A single species is at the focus of this lexical study: the hare. To narrow down the size of the data submitted to analysis, nouns and adjectives describing qualitative characteristics of the hare have been given priority; thus, common hare-denoting nouns are given emphasis. Additional areas of interest extend over terms describing its age, sex, as well as its physical and mental features. The corpus comprises lexical units drawn from $19^{\text {th }}$ - and $20^{\text {th }}$-century dictionaries of hunting lexis. It extends over present-day terms, documented in blogs and web pages frequented by hunters. The present investigation has benefited greatly from oral interviews and surveys carried out amongst Polish and Italian hunters coming from a variety of regions.
\end{abstract}

Keywords: names, adjectives, equivalent, hunting, language for special purposes 


\section{PREMESSA}

T a lingua venatoria è un sottocodice utilizzato da un gruppo sociale e professionale ben determinato, ossia dai cacciatori, dalla guardia forestale, dai boscaioli, dai veterinari, dai tecnici faunistici e da persone che si occupano della sua struttura linguistica e culturale ${ }^{1}$.

Questo linguaggio settoriale, vale a dire la lingua venatoria (o nel caso della nostra ricerca, sarebbe più opportuno parlare di lingue venatorie), costituisce una risorsa piuttosto ampia, poiché comprende termini relativi ai nomi della flora e della fauna, l'anatomia animale, le tecniche balistiche e di caccia, la cinofilia, l'equipaggiamento, i nomi professionali di cacciatori, il vecchio e nuovo ordinamento sulla caccia, l'arte culinaria e le tradizioni venatorie. La loro specificità consiste, tra le altre cose, nel fatto che il vocabolario usato dai cacciatori nel passato e nel presente non abbia subito rilevanti cambiamenti, che sono invece stati osservati nel caso di altri linguaggi specialistici. Può accadere di osservare alcuni arcaismi, ma ce ne sono relativamente meno rispetto alla lingua generale o ad altri professionaletti. I neologismi, d'altra parte, appaiono nei settori più soggetti a essere sviluppati, vale a dire nei nomi di attrezzature e nelle tecniche di caccia che diventano sempre più efficaci, e in alcuni nomi di attività venatorie.

Non vi è alcun dubbio che gli utenti di una lingua specialistica (nella fattispecie del linguaggio venatorio) siano anche quelli della lingua generale. Oggetto d'interesse dei ricercatori di lingue specialistiche sono gli elementi tipici di una determinata lingua: il socioletto (Wilkoń, 2000), con cui intendiamo il linguaggio tipico di un determinato gruppo sociale, che presenta una sua terminologia e che assolve la funzione comunicativa e cognitiva tra gli individui appartenenti a quel particolare gruppo; oppure il professioletto (ibidem) inteso come linguaggio tipico di un dato gruppo di professionisti, che presenta una sua terminolo-

1 Desidero ringraziare Alessia Mancia, Bruno Mancia, Andrea F. De Carlo, Antonio De Carlo, Marek Zdeb e Ryszard Suty, per le preziose informazioni fornitemi circa la terminologia venatoria. 
gia e che adempie alla funzione comunicativa e cognitiva all'interno di quella categoria di lavoratori.

Nel nostro lavoro di ricerca, consideriamo caratteristiche distintive del linguaggio venatorio quelle unità lessicali che sono strettamente correlate all'attività di un gruppo sociale specifico e sono tipiche di un dato socioletto. Il materiale lessicale utilizzato nella ricerca e presentato in questo articolo proviene perlopiù dai testi a stampa ${ }^{2}$ (dizionari e manuali sulla lingua e sulle tecniche di caccia, racconti editi in versione cartacea) e dall'oralità secondaria (blog, forum per i cacciatori, racconti sulla caccia pubblicati in rete). Disporre di materiale così eterogeneo presuppone anche una certa variabilità dei registri della lingua specialistica: dal livello molto formale fino a quello informale meno controllato, usato dai cacciatori in conversazioni quotidiane e durante le battute di caccia.

Riguardo alla suddivisione logica, sono state oggetto del nostro interesse la distinzione di particolari significati lessicali e la ricerca del maggior numero possibile di equivalenti esistenti nelle due lingue venatorie analizzate che riguardino una specie cacciabile come la lepre.

Questo articolo vuole essere il primo passo per la realizzazione di un futuro dizionario terminologico. Nel costruire questa parte iniziale del nostro dizionario siamo stati guidati dall'approccio onomasiologico che ci ha permesso di organizzare i lessemi in modo tematico, evitando così quello alfabetico. Questo approccio, inoltre, ci ha consentito di valutare - dove era possibile - l'equivalenza tra i termini delle due lingue qui analizzate. Considerata la mole di materiale, in questo articolo ci siamo limitati all'analisi semantica dei nomi e degli aggettivi qualificativi legati alla lepre. La terminologia scelta è stata suddivisa in base ai seguenti gruppi: 1. nomi di specie e di singoli animali su esempio della lepre: a) nomi che si riferiscono alla specie o ai singoli animali, b) nomi che si riferiscono alle abitudini o ai luoghi di vita della lepre; 2. nomi che indicano il sesso; 3 . nomi che indicano l'età; 4. nomi e aggettivi indicanti i tratti distintivi di una lepre.

2 In bibliografia forniamo un elenco completo delle fonti in base alle quali è stato creato il corpus lessicale. 


\section{NOMI DI SPECIE E DI SINGOLI ANIMALI SU ESEMPIO DELLA LEPRE}

2.1. Nomi che si riferiscono alla specie o ai singoli animali:

In Polonia esistono due specie di lepre, i cui nomi ufficiali completi sono:

1. Lepre comune o lepre europea (Lepus europaeus; in polacco zając szarak) - è la specie di lepre più diffusa in tutta la Polonia ad eccezione dei monti Tatra e dei Sudeti. Questa è l'unica specie di lepre che può essere cacciata in Polonia;

2. Lepre variabile o lepre bianca (Lepus timidus; in polacco zając bielak) - specie rara, esiste solo nel Nord-Est della Polonia; genere di lepre bianca; si tratta di una specie protetta.

In Italia invece ci sono quattro varietà (Marzola, 2013; Casanova, Capaccioli \& Cellini, 1993):

1. Lepre europea (Lepus europaeus) - questa specie presenta una distribuzione estesa in tutta l'Italia peninsulare; caratterizzata dalla pelliccia costituita da giarra e borra, si presenta in genere di colore fulvo-grigio più scuro sul dorso, nuca e testa;

2. Lepre italica (Lepus corsicanus) - è una specie diffusa unicamente nell'Italia centro-meridionale; si caratterizza dalle dimensioni inferiori rispetto alla lepre europea e dal mantello più rosso su tutto il dorso e sulle zampe, mentre nel sottopancia è d'un bianco candido;

3. Lepre sarda (Lepus capensis mediterraneus) - è un lagomorfo molto simile alla lepre comune o europea, anche se più piccola, tipica della Sardegna in cui è stata introdotta dall'Africa; il colore del mantello è un misto di nero e fulvo che la rendono quasi a macchie, mentre l'interno degli arti non è bianco, ma di una tonalità simile a quella esterna; di abitudini crepuscolari e mattutine, la lepre sarda rispetto all'europea ha un'andatura più lenta e un maggior attaccamento al territorio in cui vive;

4. Lepre variabile (Lepus timidus) - è una sottospecie presente nelle zone alpine a un'altitudine superiore ai 1200 metri, con una pelliccia bianca nella stagione invernale. 
Tra i leporiti sopraindicati tre appartengono alle specie cacciabili: la lepre europea, la lepre variabile e la lepre sarda. L'autoctona lepre italica, a causa della riduzione del suo habitat, dei veleni impiegati in agricoltura e della competizione con altre specie, è praticamente quasi scomparsa. È ancora cacciabile soltanto in Sicilia (nel periodo compreso tra il 15 ottobre e il 30 novembre).

Il carattere tassonomico della zoologia venatoria (e le altre discipline scientifiche) impone rigide applicazioni dei meccanismi di formazione di parole il cui obiettivo è "quello di ottenere una nomenclatura sempre più precisa e semplice, utilizzabile dagli studiosi di tutti i paesi. [...] Ciò non impedisce che, accanto alla nomenclatura ufficiale, rigorosamente stabilita dagli organismi preposti a tale scopo e codificata in italiano [...], si sviluppino o si mantengano denominazioni alternative, proprie delle diverse scuole e tradizioni" (Bianco, 2004, p. 592). Sia in polacco che in italiano osserviamo il fenomeno di una doppia denominazione: una in lingua latina, in quanto considerata la lingua della scienza, e un'altra in lingua nazionale.

Nelle fonti polacche puramente scientifiche abbiamo a che fare con una denominazione omogenea: vale a dire, un doppio sintagma nominale in polacco $(\mathrm{N}+\mathrm{N}$ : zajac szarak, zajac bielak $)$ e un nome latino composto da due parti ( $\mathrm{N}+$ Agg: Lepus europaeus e Lepus timidus). $\mathrm{Nel}$ caso dei materiali di caccia molto dipende dal registro linguistico. Il nome completo polacco e latino è ravvisabile soltanto in testi molto formali, contenenti disposizioni di legge, definizioni ecc. (si vedano per esempio le leggi venatorie e il manuale di zoologia venatoria, oppure l'enciclopedia di specie cacciabili). Oltre alle fonti già menzionate possiamo incontrare nomi semplificati di elementi singoli tanto nella lingua scritta (manuali di tecnica venatoria, dizionari della lingua e cultura venatoria, stampa venatoria, racconti dei cacciatori pubblicati in versione cartacea e in rete), quanto nell'oralità secondaria (blog, forum dei cacciatori) che nelle semplici conversazioni degli specialisti: cacciatori, silvicoltori, zootecnici. Ciò avviene dunque:

1) in riferimento alla specie:

a) zając: è un caso di spostamento semantico - sineddoche: il nome generico, comune viene usato solo nel caso della lepre 
europea in base alle leggi venatorie e alla situazione ecologica visto che "zając szarak" è l'unica specie cacciabile in Polonia. Come osserva Władysław Dynak (1993, p. 175):

[...] owo zoologiczno-łowieckie rozróżnienie (szarak vs. bielak), choć ma pokrycie w rzeczywistości, nie znajduje jednak odzwierciedlenia w powszechnej praktyce językowej. Powód jest oczywisty - terytorialny zasięg występowania obu gatunków: w wypadku szaraka pokrywający się całkowicie z zasięgiem polszczyzny, bielaka jedynie w znikomym stopniu. ${ }^{3}$

b) szarak: si tratta di un caso di ellissi: il significato di tutto il sintagma $(\mathrm{N}+\mathrm{N})$ viene trasferito al secondo elemento nominale;

c) bielak: come sopra;

2) come forme sinonimiche - le definizioni generali della lepre. In pratica, la più comune è la lepre europea perché è l'unica specie cacciabile in Polonia:

a) kopyra: alcune fonti considerano questo nome come regionale o dialettale (Hoppe, 1980, p. 89; Krzemień, 1984, p. 72), ma osservando le pubblicazioni venatorie contemporanee ${ }^{4}$ si evince che il nome 'kopyra' è usato in modo intercambiabile con il nome 'szarak'. Ciò significa che questa forma non è limitata territorialmente ed è contemporaneamente usata dai cacciatori in tutta la Polonia sia in forma scritta che parlata. Trattiamo il termine 'zając szarak', 'szarak' come sinonimo specialistico, venatorio. I nostri risultati hanno confermato l'analisi di Stanisław Hoppe (1966, p. 61), proposta nel suo dizionario della lingua venatoria. Tuttavia, nella prima edizione degli anni Sessanta il termine 'kopyra' (1966, p. 61) è accompagnato da

3 [...] questa distinzione venatorio-zoologica [szarak (europea) vs bielak (bianca)], sebbene abbia un riscontro nella realtà, non si riflette nella pratica linguistica comune. La ragione è ovvia: il limite dell'area territoriale di entrambe le specie: nel caso della lepre europea copre completamente i limiti della lingua polacca, mentre la lepre bianca - solo in misura trascurabile (trad. nostra).

4 Si tratta qui di due principali mensili Łowiec Polski e Brać Łowiecka pubblicati negli anni 2015-2018. 
una definizione dialettale, che invece scompare nella terza edizione estesa e corretta del 1981. Questo ci dà ragione di credere che le varietà diatopiche, diamesiche e diafasiche spostano e ampliano l'ambito di utilizzo del lessema 'kopyra' rispetto alla varietà regionale, ossia il suo impiego viene esteso tanto al parlato più trascurato e informale, quanto all'uso della lingua venatoria standard, nonché allo scritto;

b) kot: termine colloquiale, diffuso su tutto il territorio nazionale, usato spesso dai cacciatori per indicare una lepre assai vecchia, grande, veloce e astuta.

$\mathrm{Ci}$ sembra che anche le forme menzionate, che descrivono la lepre nei seguenti punti, debbano essere definite termini regionali - forme sinonimiche il cui uso è geograficamente limitato. Purtroppo, in questa fase della ricerca non indichiamo in dettaglio la portata geografica del loro uso perché ciò richiederebbe ulteriori ricerche, per cui ci riserviamo di trattarla in eventuali articoli futuri. Pare che sotto la nozione polacca di "uso dialettale" si nascondano lessemi usati per i nomi del registro più basso, meno formale, più popolare e usato nel parlato e nello scritto, spesso solo in una certa area o regione.

Dopo l'analisi di dizionari e di manuali di lingua e cultura venatoria polacca del XIX (Kozłowski, 1822) e del XX secolo (Hoppe, 1966, 1980, 1981; Pasławski, 1973; Krzemień, 1984; Żeromski, 1990; Biały \& Jóźniak, 1994), nonché delle pagine on line di PZŁ e di altri circoli venatori (Poradnik Łowiecki, 2019; Klimowicz, 2017) abbiamo individuato (Malczewska \& Smoła, 2018; Smoła, 2017) altri 14 sostantivi che indicano la lepre, usati in modo interscambiabile con il nome 'szarak' a seconda della regione, che nel linguaggio settoriale italiano definiamo geosinonimi, cioè "sinonimi a distribuzione geografica complementare" (Sobrero \& Miglietta, 2006, p. 81): korpal, koszlon, kaszlon, wacho, skotak, ślepak, śpioch, koczkon, jepur, wytrzeszczak, filip, kusy, siwak, michałek nonché un nome che indica il suo habitat, ovvero: poluch una lepre a cui piace stare nei campi.

La maggior parte di questi nomi è nota solo a un ristretto gruppo di specialisti, perlopiù cacciatori, e anche in misura limitata - cioè conoscono il nome, ma non lo usano. 
Il nome filip è usato anche nella lingua comune grazie a un detto: "wyskoczyć jak filip z konopi". La maggior parte dei polacchi conosce e usa questo modo di dire, ma non associa sempre il nome filip al termine venatorio 'lepre' che, secondo Władysław Dynak (1993, p. 176), proverrebbe dalla Bielorussia dove è invece un sinonimo di caccia alquanto popolare. La pratica linguistica sembra confermarlo, dato che i cacciatori danno questa variante come forma più usata dagli altri cacciatori nella regione della Podlachia.

Oltre ai suddetti sinonimi, Wiktor Kozłowski (1822, p. 91) offre un'altra forma riguardante solo la lepre bianca: l'arcaismo siniak, che proviene dai tempi della Confederazione polacco-lituana. 'Siniak' indica la lepre bianca nel periodo della muta (il cambio della pelliccia della lepre, che passa da quella bianca invernale alla grigia estiva, dove i peli bianchi cadono e appaiono quelli grigi) e sembra un cambiamento di colore dal bianco al blu e infine al grigio. È anche il termine 'bielak', che non cambia l'intera pelliccia d'inverno, ma solo sbianca sul ventre e sui fianchi. Attualmente il nome è poco utilizzato nel contesto della lepre (che deriva dal divieto in Polonia di cacciare le lepri bianche; da qui il raro utilizzo di questo lessema in fonti e conversazioni specialistiche) e non troviamo tale significato nei dizionari di lingua venatoria, sebbene la nostra ricerca mostri che il termine è generalmente noto a un gremito gruppo di cacciatori.

Nelle fonti scientifiche popolari, ad esempio nei blog sulla natura che sono indirizzati a una vasta gamma di destinatari, possiamo imbatterci in un termine suggestivo come $d$ tugouchy ${ }^{5}$ (orecchie lunghe), derivato dalle tipiche orecchie lunghe della lepre, conosciute in polacco come stuchy. Questo non è affatto un termine specialistico.

Analizzando diverse fonti scritte italiane (dizionari della lingua e cultura venatoria, stampe e manuali di tecnica venatoria, manuali di

5 Un esempio di contesto d'uso: "Nie zabierajcie małych zajęcy ze środowiska!!! Warto też pamiętać, że odchowanie takiego malucha jest bardzo trudne. Wymaga on wysokokalorycznego mleka i niekoniecznie będzie od razu chętnie jadł. Oczywiście można na nie patrzeć, nie płoszyć i pod żadnym pozorem nie dotykać. Trzeba się przy takim spotkaniu spokojnie wycofać i dać dtugouchemu czas na zniknięcie w suchej trawie" (Konieczny, 2019). 
zoologia venatoria, storie di caccia pubblicati in versione cartacea ecc.) e di oralità secondaria (blog, forum dei cacciatori, video-chat, racconti dei cacciatori pubblicati in rete ecc.) si osserva che i cacciatori, zoologisti, tassidermisti, silvicoltori, tecnici faunistici, nonché altri conoscitori e appassionati della cultura venatoria usano molti nomi più popolari e semplificati per riferirsi ai leporiti.

Di seguito vorremmo presentare e discutere brevemente tutte le forme riscontrate. In totale, sono stati analizzati 12 lessemi provenienti da diversi materiali ${ }^{6}$ con registri differenti: dal registro settoriale formale, passando per la lingua specialistica "standard", fino all'italiano settoriale usato nelle conversazioni - una varietà informale altamente trascurata. Gli altri nomi che si riferiscono alle specie dei leporiti nella lingua venatoria italiana sono i seguenti:

a) per indicare la lepre europea (Lepus europaeus) ritroviamo tre sintagmi: N + Agg: lepre comune, lepre grigia, lepre bruna nonché il nome generale lepre, che si evince dalla sua diffusione ed è il più riconoscibile di questa specie di lepre;

b) per indicare la lepre variabile (Lepus timidus) esistono due altre denominazioni: lepre bianca e lepre alpina;

c) riguardo alla lepre italica (Lepus corsicanus) nei testi e nei discorsi analizzati si può riscontrare un solo caso di ellissi, quando il sintagma viene trasferito al solo aggettivo. Si osserva infatti il processo di conversione con la trasposizione del lessema italica a un nome deaggettivale: la lepre italica $\rightarrow$ l'italica, cioè [italica] Aggettivo $\rightarrow\left(1^{\prime}\right)$ [italica $]_{\text {Nome }}$.

I termini summenzionati (eccetto il termine lepre italica), a differenza della terminologia polacca, non costituiscono nomi semplificati o nomi colloquiali, ma sono nomi usati in modo intercambiabile con lepre europea, lepre variabile. Ciò è testimoniato dal contesto e dalle fonti da cui provengono le forme citate, per esempio il termine scelto e usato in base al principio dell'antinomia: se l'interlocutore ha usato il nome che connota il colore del pelo della lepre bianca, avviene spesso in prospettiva comparativa rispetto alle specie di lepre europea e allo-

${ }^{6}$ L'elenco completo è riportato in bibliografia. 
ra ha scelto il sintagma con l'aggettivo di colore lepre bruna o lepre grigia. Invece, il termine lepre alpina si trova più spesso nei manuali di arte venatoria pubblicati nel Nord Italia, sulle Alpi: per esempio in Trentino-Alto Adige. Forse si tratta di una scelta consapevole e l'uso di elementi toponimici nella struttura del sintagma nominale è una forma di identificazione del mittente con un dato termine. Questo è un modo per mostrare l'appartenenza di questa specie di lepre alla comunità dei cacciatori di montagna.

Oltre ai nomi composti da due parti che descrivono la lepre annotatati principalmente nei registri alti delle lingue, nelle dichiarazioni scritte e orali dei cacciatori troviamo anche altre forme sinonimiche di un elemento che descrivono la lepre: orecchiona, leprone, lagomorfa, baffona ${ }^{7}$, roditore.

Il nome orecchiona è una forma che compare nel registro scritto neo-standard e nel registro orale - informale dei cacciatori. Abbiamo osservato il suo uso nei racconti dei cacciatori pubblicati nei libri, ma anche in rete (blog, forum). È una forma usata per non ripetere il nome lepre. Osservando la sua frequenza d'uso e il contesto conversativo, si può concludere che l'equivalente polacco di orecchiona è kopyra, che si presenta con una certa frequenza e nella stessa varietà, si tratta di un termine trasmesso soprattutto oralmente, anche se in una traduzione letterale sarebbe dtugouchy. La differenza, tuttavia, consiste nel fatto che l'orecchiona è un termine dal colorito "linguistico" degli stessi cacciatori; il termine, secondo i cacciatori polacchi, è considerato molto più colloquiale di kopyra come evidenziato dalla punteggiatura. Nei testi analizzati, il termine orechiona era sempre citato tra caporali, il che significa che gli specialisti stessi non sono completamente convinti del carattere lessicalizzato del termine trattandolo piuttosto metonimicamente. A nostro parere, il termine summenzionato andrebbe considerato come un neologismo specialistico e, di conseguenza, andrebbe incluso

7 Esempio d'uso: "Qualche volta la [lepre] caccio da solo, senza il cane, in assoluto silenzio con il mio inseparabile "ventino" ed è ancora più emozionante perché sono io che la devo scovare... È una sfida tra me e l'astuta baffona, molte volte vince Lei, qualche altra vinco io, ma ogni volta è sempre una grande indimenticabile emozione..." (Calibro, 2012). 
nelle risorse lessicografiche. Tenendo presente la frequenza e il contesto d'uso come criterio principale, il sostantivo orecchiona soddisfa pienamente questa ipotesi.

Il termine leprone, che si incontra nelle conversazioni e nei racconti, è un alterato vezzeggiativo-accrescitivo, dal momento che serve sia a descrivere una lepre di dimensioni insolitamente grandi sia a esprimere altre sue qualità: per esempio, l'astuzia, la velocità e l'ottimo istinto. Una simile derivazione connota positivamente questa forma alterata, poiché, essendo frequente nelle conversazioni e nei racconti editi, serve ai cacciatori per vantarsi di una battuta di caccia avvenuta con successo o semplicemente riferire il loro piacere nell'osservare un bellissimo o magnifico esemplare di questa specie.

Il termine leprone ha anche un secondo significato specialistico: connota il maschio della lepre. Il suo equivalente in polacco è gach.

Nei testi analizzati si trova anche l'espressione lagomorfa. In zoologia il termine 'lagomorfi' è stato definito nel 1912 e indica 'in ordine dei mammiferi terrestri erbivori, a cui appartengono le famiglie leporidi e ocotonidi, caratterizzati dalla presenza di due coppie di incisivi, di cui la prima molto allungata e la seconda piccola e posta dietro di questa (...)" (Treccani, 2019). Le due famiglie sono entrambe storicamente diffuse in Italia ma, mentre l'unico rappresentante degli Ocotonidi (Prolagus sardus) risulta essere estinto da più di due secoli, la famiglia dei Leporidi è attualmente diffusa nel territorio ed è rappresentata da tre generi (Lepus, Oryctolagus e Sylvilagus) e cinque specie (lepre europea, lepre italica, lepre variabile, lepre sarda, coniglio selvatico e silvilago) (Zennaro, 2014, p. 9).

Troviamo il lessema lagomorfa nei racconti e sui forum on line. Qui abbiamo a che fare con un caso di sineddoche: la sostituzione di un nome comune 'lepre' con un nome proprio zoologico, che prevede la simultanea restrizione di significato della parola 'lagomorfa' dall'ordine dei mammiferi, un nome di un genere (Lepus) e/o un nome di una specie (lepre bruna, bianca o sarda).

I materiali analizzati dell'oralità secondaria includevano anche un termine spiritoso e suggestivo baffona, che fa riferimento ai peli sensoriali della lepre. Questi ricordano proprio dei baffi. Oltre a esso, pos- 
siamo ricordare il lessema roditore usato per non ripetere il nome comune 'lepre'. Consideriamo entrambi questi nomi come occasionalismi (cf. Treccani on line), perché sono apparsi negli enunciati di due cacciatori e al di fuori del loro contesto d'uso è difficile affermare se abbiamo a che fare con sinonimi di lepre o con un altro animale.

2.2. Nomi che si riferiscono alle abitudini e all'ambiente naturale della lepre

Oltre ai nomi sinonimici generali della lepre già elencati, nel dizionario della lingua venatoria degli anni Quaranta troviamo cinque termini importanti che indicano il suo ambiente e la ricerca di cibo, vale a dire:

a) lepre ladra: "la lepre che vive presso o nelle paludi" (Farini \& Ascari, 1941, p. 367);

b) lepre stanziale: "la lepre che rimane sempre nello stesso paese" (ibidem);

c) lepre vagante: "la lepre che cambia paese. Questo fa il maschio, specie quando è perseguitato" (ibidem);

d) lepre di montagna: "la lepre che vive ne' colli. Ma dicesi anche de' maschi solinghi, i quali si stanziano nelle parti più alte dei monti, e sono i più ammalizziti e scaltri a sottrarsi al cacciatore" (ibidem);

e) lepre di pianura: "quella de' piani, che prende caratteri alquanto diversi de la precedente, e ne è più grande e in certi casi migliore a mangiarsi" (ibidem).

Per riassumere questa parte, possiamo affermare che, oltre ai nomi ufficiali polacchi e latini della lepre, esistono 22 termini descrittivi in polacco, tra cui 4 termini sinonimici generici, 2 sinonimi senza specificare la specie al livello nazionale, 14 geosinonimi e un sinonimo metaforico di lepre ("lunghe orecchie") usato dai non cacciatori. In italiano, tuttavia, a parte i nomi ufficiali polacchi e latini della lepre, troviamo 17 definizioni di lepre, di cui 7 sono sinonimi relativi alla specie, 5 sono definizione di lepre in base al suo ambiente naturale e 5 sinonimi generali, due dei quali vengono trattati come occasionalismi. 


\section{NOMI INDICANTI IL SESSO}

Nella seguente tabella presentiamo un elenco di lessemi indicanti il sesso di una lepre:

Tabella 1: Nomi indicanti il sesso

\begin{tabular}{c|c|c}
\hline Lingua polacca & Lingua italiana & Significato \\
\hline gach & leprone & 'maschio della lepre' \\
\hline $\begin{array}{c}\text { kocica } \\
z a j e ̨ c z y c a\end{array}$ & - & 'femmina della lepre' \\
\hline
\end{tabular}

Riguardo ai nomi che si riferiscono al sesso dell'animale, nella lingua venatoria polacca esistono 2 lessemi (gach e kocica) che indicano chiaramente le differenze di sesso. Viene anche usato il termine kot che nel contesto può indicare il sesso del maschio, ma è anche utilizzato come sinonimo di una lepre adulta come specie di caccia. Nei dizionari troviamo anche un termine tratto dalla lingua generale: zajęczyca; in pratica, è la forma più usata dai cacciatori. La lingua italiana presenta una situazione singolare, ossia esiste il maschile leprone, mentre per indicare il sesso della femmina si ricorre al nome di genere promiscuo, alla parafrasi 'femmina della lepre' oppure 'la lepre femmina'. Il termine 'leprone' ha altre connotazioni: può indicare altresì una lepre dalle grandi dimensioni, ormai matura, esperta e scaltra. Ciò è forse dovuto al fatto che la lepre non presenta dimorfismo sessuale e il nome generico lepre è di genere femminile. Pertanto, nella lingua italiana, in modo naturale, spontaneo e intuitivo la lepre viene associata alla "femminilità".

\section{NOMI INDICANTI L'ETÀ}

Nella seguente tabella presentiamo un elenco di lessemi indicanti l'età e il periodo di nascita di una lepre: 
TABELla 2: Nomi indicanti l'età

\begin{tabular}{|c|c|c|}
\hline Lingua polacca & Lingua italiana & Significato \\
\hline $\begin{array}{l}\text { kocie } \\
\text { kociak }\end{array}$ & $\begin{array}{c}\text { leprotto } \\
\text { lepracchiotto } \\
\text { lepratto } \\
\text { leproncello }\end{array}$ & 'giovane lepre' \\
\hline marczak & - & $\begin{array}{c}\text { 'giovane lepre nata nel mese } \\
\text { di marzo' }\end{array}$ \\
\hline $\begin{array}{l}\text { wrześniak } \\
\text { nazimek }\end{array}$ & - & $\begin{array}{c}\text { 'giovane lepre nata in settembre, } \\
\text { in autunno' }\end{array}$ \\
\hline$\dot{z} a k$ & - & 'giovane lepre inesperta' \\
\hline
\end{tabular}

In polacco esistono due termini più generali per indicare una lepre giovane con il suffisso neutro -e (kocie) e con il suffisso -ak (kociak). $\grave{E}$ un suffisso diminutivo che nella lingua generale funziona negli alterati denominali della base con il suffisso $-e$ (kocię $\rightarrow$ kociak) ed è caratterizzato da colloquialità e trattato come una variante regionale utilizzata nella Polonia centrale e settentrionale. Nel caso della lingua venatoria, è assente una relazione affettiva. Entrambi i nomi sono termini specifici usati in modo intercambiabile come sinonimi.

La lingua italiana, d'altra parte, è ricca di forme sinonimiche che si riferiscono alla lepre appena nata. L'origine di ciò è ravvisabile nella stessa morfologia della lingua italiana che si caratterizza dalla ricchezza di formule alterative, dalla varietà e dalla grande quantità di diminutivi. Per tale motivo si osservano quattro nomi per indicare una giovane lepre. La forma lepratto (piccola lepre, leproncello) si incontra molto raramente, oggi perlopiù sostituita dalla forma più moderna leprotto. I dizionari Farni e Ascari (1941, p. 369) la indicano come forma letteraria e non venatoria. Finora, nelle nostre ricerche sulla terminologia specialistica non ci è capitato di incontrare questo termine.

Non esiste alcun alterato diminutivo con il suffisso particolarmente produttivo -ino, dal momento che le forme con questo suffisso funzionano come aggettivi denominali specialistici, si pensi a leprino/leporino, cervino, volpino ecc. 
Un fenomeno interessante si verifica nel caso di quei lessemi che si riferiscono al periodo riproduttivo. Nella lingua di caccia polacca sono state definite lessicalmente la prima e l'ultima cucciolata (marczak, wrześniak/nazimek). Nei dizionari venatori italiani invece non si riscontrano nomi che si riferiscono ai periodi riproduttivi.

\section{NOMI E AGGETTIVI INDICANTI I TRATTI DISTINTIVI DI UNA LEPRE}

Nella seguente tabella presentiamo l'elenco di nomi e aggettivi indicanti i tratti distintivi del carattere della lepre: astuzia, abilità e velocità.

TABella 3: Nomi e aggettivi indicanti i tratti distintivi di una lepre

\begin{tabular}{|c|c|c|c|}
\hline \multicolumn{2}{|c|}{ Lingua polacca } & \multicolumn{2}{|c|}{ Lingua italiana } \\
\hline Nome & Significato & Nome & Significato \\
\hline gracz & $\begin{array}{l}\text { 'animale esperto, astuto, } \\
\text { saggio, capace di abbindo- } \\
\text { lare cani e cacciatori' }\end{array}$ & leprone & $\begin{array}{l}\text { 'vecchia lepre, esper- } \\
\text { ta e scaltra' }\end{array}$ \\
\hline $\begin{array}{l}\text { defilator } \\
\text { (nome) }\end{array}$ & $\begin{array}{l}\text { sostantivo usato (per } \\
\text { riferirsi alla lepre e/o al } \\
\text { cinghiale, talvolta anche } \\
\text { al fagiano oppure ad altri } \\
\text { uccelli) quando la preda at- } \\
\text { traversa parallelamente alla } \\
\text { mira dei cacciatori e alcuni } \\
\text { di essi sparano spesso con } \\
\text { scarso risultato e allora si } \\
\text { dice che tutti hanno sparato } \\
\text { al defilator (scanato) }\end{array}$ & $\begin{array}{c}\text { scanato/a (nome } \\
\text { o aggettivo) }\end{array}$ & $\begin{array}{l}\text { 'animale (general- } \\
\text { mente cinghiale o le- } \\
\text { pre) che arriva alla } \\
\text { posta senza essere } \\
\text { inseguito dai segugi' }\end{array}$ \\
\hline skromy & grasso & - & - \\
\hline nieskromny & magro & - & - \\
\hline staby & magro, debole & - & - \\
\hline spadty & magro, debole & - & - \\
\hline chybki & agile e veloce & - & - \\
\hline
\end{tabular}


Per quanto riguarda i termini che si riferiscono al carattere della lepre, essi sono generalmente condivisi da entrambe le lingue analizzate. La lepre è astuta, scaltra, saggia e velocissima. Tuttavia, tutte queste caratteristiche menzionate sono espresse con l'aiuto di aggettivi che sono usati e conosciuti anche nella lingua generale.

In lingua italiana esiste solo un termine specialistico usato sia come nome sia come aggettivo: scanato, con cui si indica sia la lepre che il cinghiale, che udendo l'abbaiare dei cani oppure senza esser inseguiti dai segugi, escono dai covi e sfilano davanti ai cacciatori. Questi sparano alla lepre, che nel loro idioletto è definita con l'espressione "lepre scanata", ma spesso "padellano", ossia falliscono il colpo. Nella lingua venatoria polacca abbiamo un termine equivalente preso dal gergo militare: defilator, che deriva dal verbo defilować, cioè 'marciare, sfilare'. Si utilizza questo termine per indicare la cacciagione (più spesso la lepre, ma anche il cinghiale e il fagiano) che appare direttamente di fronte alla mira dei cacciatori come se volesse sfilare. Il termine defilator indica anche la selvaggina che è sfuggita sana e salva dopo una simile "parata" davanti ai cacciatori. In italiano viene dato il contesto di questa sfilata davanti ai cacciatori e/o ai cani, che è ovviamente dipende dai realia extralinguistici: in Polonia è vietato cacciare una lepre con l'uso dei cani.

In lingua polacca esiste anche un altro sostantivo per indicare una lepre astuta, veloce, esperta, ossia gracz, il cui corrispondente italiano potrebbe essere leprone. Tuttavia, questo termine ha un significato più ampio: può riferirsi alle dimensioni fisiche e al peso della lepre (grande, grassa, adulta - in polacco kot); può significare il maschio della lepre (il corrispettivo polacco è allora gach) oppure può riferirsi a caratteristiche mentali legate alla predisposizione fisica (astuzia, scaltrezza, velocità) e allora il suo corrispettivo è gracz.

Nella lingua dei cacciatori polacchi si incontrano anche cinque aggettivi qualificativi: skromny per indicare lepre grande e grassa. $\grave{E}$ un aggettivo denominale che deriva dal termine strettamente venatorio skrom che indica il grasso di lepre. Inoltre, abbiamo anche tre forme per caratterizzare la lepre magra, cioè nieskromy - un antonimo dell'aggettivo già citato e in più staby e spadty. I cacciatori usano 
anche un aggettivo specialistico per sottolineare una grande velocità e abilità: chybki.

Stanisław Hoppe (1980, p. 93) nel suo libro Polski język łowiecki ("La lingua polacca venatoria", 1980) afferma che il termine si applica solo al coniglio, dal momento che esso ha "i sensi più perfetti della lepre, e spesso è più veloce di essa" (1980, p. 93). Attualmente il termine ha un significato più ampio. I cacciatori intervistati durante la nostra ricerca hanno dichiarato chiaramente di usare questo termine prima di tutto in riferimento alla lepre, poiché nella realtà venatoria è un animale molto veloce e agile, capace di confondere i cacciatori.

In italiano, tuttavia, a parte il termine scanato, non abbiamo notato nessun altro aggettivo specialistico che caratterizzi gli attributi di una lepre.

\section{CONCLUSIONI}

Il materiale fattografico della lingua polacca e italiana ci ha permesso di distinguere 62 lessemi, che nella lingua di caccia riguardano i sinonimi generali di un animale, la specie, i nomi delle lepri legate all'ambiente in cui vivono, l'età, il sesso e le caratteristiche tipiche. Ci sono 38 espressioni di lepre nella lingua di caccia polacca, di cui 33 nomi e 5 aggettivi qualificativi; in italiano distinguiamo 24 termini, di cui 23 sono nomi oppure sintagmi nominali e anche il termine scanato che assolve il ruolo di nome oppure aggettivo qualificativo.

Le nostre indagini mostrano un vantaggio quantitativo (risulta da un numero di termini che includono anche forme sinonimiche) e qualificativo (la ricchezza numerica delle espressioni e dei campi semantici) dei lessemi della lingua polacca rispetto a quelli della lingua di caccia italiana. La lingua italiana, in generale, è concettualmente più povera (solo il 40\% dei termini analizzati sono italiani), ma ha ben quattro diminutivi che si riferiscono alla lepre giovane, molti dei quali sono dovuti essenzialmente alla natura della lingua italiana ricca di nomi derivati e alterati.

Questa situazione lessicale si deve a due motivi. In Polonia, la lingua di caccia è una parte importante della cultura venatoria, ogni cacciatore 
deve conoscerla ed essere in grado di usarla in modo fluente. Parlarla significa conoscere tutti i rami di questa cultura, inclusa una descrizione della selvaggina. In Italia invece, la conoscenza della lingua di caccia non è richiesta nella comunità dei cacciatori. I cacciatori italiani usano questo linguaggio molto meno rispetto ai polacchi, il che probabilmente contribuisce a una più debole divulgazione nonché al suo sviluppo più lento.

In Italia la cultura della caccia alla lepre si differenzia in modo significativo da quella polacca. La caccia alla lepre sulla Penisola è molto popolare, e gli stessi lepraioli e segugisti sono considerati fra i migliori cacciatori. La caccia alla lepre è appassionante e abbastanza diffusa, anche perché sono accettati metodi di caccia che in altri paesi (compresa la Polonia) sono proibiti. In generale, tenendo conto della quantità di articoli apparsi sulla stampa specialistica ${ }^{8}$, delle informazioni contenute sulle pagine Internet dei circoli venatori e della Federazione della Caccia Italiana, nonché delle affermazioni degli stessi cacciatori, si può constatare che la lepre nella caccia italiana occupa una posizione di primo piano e la caccia alla lepre porta fama e rispetto al cacciatore. La lepre è un animale molto veloce e agile, quindi la caccia alla lepre è estremamente difficile, richiede pazienza, grande senso di osservazione, un certo istinto innato e buoni segugi; è un'arte venatoria raffinata. Questo si può riscontrare anche a livello del lessico specialistico legato alla praticità. Dalle nostre ricerche si evince che la lingua di caccia italiana ha relativamente pochi sinonimi per indicare la lepre, ma possiede invece molti termini che descrivono il comportamento della selvaggina durante la fuga e la caccia stessa. Ciò testimonia il pragmatismo dei cacciatori: l'arte di osservare, da cui deriva la precisione lessicale dei movimenti della lepre (vale a dire, tutti i termini relativi ai salti, alle fughe e ai movimenti sul campo). Tutto questo contribuisce al successo della caccia. La questione più importante è l'organizzazione della caccia, che deve essere il più possibile efficace, e il suo corso. Il comportamento della lepre è in secondo piano, per cui non c'è un'urgenza nel determinare linguisticamente le sue parti anatomiche o i suoi comportamenti.

\footnotetext{
${ }^{8}$ L'elenco completo di fonti on line e riviste viene riportato in bibliografia.
} 
Un'altra ragione della "limitatezza" della terminologia venatoria italiana è la situazione sociolinguistica del Paese: la presenza del bilinguismo e della diglossia (ossia, una varietà linguistica alta per gli usi scritti e formali, e una varietà bassa per gli usi parlati informali). In Italia, il dialetto viene spesso utilizzato durante le conversazioni con amici e familiari, che, a differenza della Polonia, è uno strumento vivo di comunicazione tra i propri cari. Da numerose conversazioni con i cacciatori italiani risulta che durante la caccia, gli incontri e le relazioni orali - in generale durante la comunicazione quotidiana tra specialisti di un club di caccia si usa molto spesso il dialetto locale e non la lingua italiana specialistica, che non ha una tradizione secolare come quella polacca ${ }^{10}$. Di conseguenza, la lingua venatoria italiana ha avuto meno probabilità di sviluppare, diffondere o introdurre nuovi termini. I lessemi che abbiamo identificato nel corso della nostra ricerca sono espressioni usate dai cacciatori nella comunicazione sia scritta che orale, dove ci sono persone di diverse località o regioni nei luoghi pubblici. Molti cacciatori, in particolare i più anziani e più esperti, che ci forniscono un valido aiuto nel lavoro lessicografico, confermano che spesso usano il dialetto per parlare di caccia.

Generalmente la terminologia venatoria (specialmente quella polacca) legata alla lepre è molto ampia e contiene lessemi precisi, univoci che indicano l'età, il sesso, le caratteristiche fisiche (la capacità di muoversi rapidamente) o psicologiche che sottolineano l'astuzia, l'istinto e l'intelligenza innata dell'animale. Inoltre, nelle conversazioni, nelle storie di caccia e nei manuali di arte venatoria, i cacciatori ricorrono a molte forme sinonimiche per descrivere le specie di lepri che vivono nei loro paesi.

9 Ma solo in relazione ai nomi associati alla specie, ai singoli animali, al sesso, all'età e ai tratti del carattere della lepre. In questo articolo non abbiamo analizzato i lessemi legati all'anatomia, ai movimenti, alla capacità di fuga della lepre, neanche i nomi di caccia e cacciatori di lepri, che sono relativamente numerosi.

10 Ricordiamo che "negli anni dell'unificazione nazionale attorno il 1861, lungi dal rappresentare la totalità dei cittadini italiani, erano poco più di seicentomila su una popolazione che aveva superato i 25 milioni di individui: [...]: dunque, il 2,5\% della popolazione" (De Mauro, 2001, p. 43). 


\section{BIBLIOGRAFIA}

Bianco, F. (2004). Formazione delle parole nelle terminologie tecnicoscientifiche. Botanica e zoologia. In M. Grossman \& F. Rainer (Eds.), La formazione delle parole in italiano (pp. 591-597). Tübingen: Niemeyer.

De Mauro, T. (2001). Storia linguistica dell' Italia unita. Roma: Laterza.

Dynak, W. (1993). Łowy, łowcy i zwierzyna w przysłowiach polskich. Wrocław: Towarzystwo Przyjaciół Polonistyki Wrocławskiej.

Hoppe, S. (1980). Polski język towiecki. Warszawa: Państwowe Wydawnictwo Rolnicze i Leśne.

Malczewska, B., \& Smoła, J. (2018). La lepre nella terminologia venatoria polacca, russa e italiana: un compendio lessicale. In E. Nikolajová Kupferschmidtová \& P. Prando Šušová (Eds.), Rebus linguae vol. 2 (pp. 8-36). Bratislava: Comenius University of Bratislava. Faculty of Medicine.

Smoła, J. (2017). Zając w polskiej i włoskiej terminologii łowieckiej. In B. Borkowska-Kępska \& G. Gwóźdź (Eds.), LSP Perspectives 2. Języki specjalistyczne - nowe perspektywy 2. Dąbrowa Górnicza: Wydawnictwo Wyższej Szkoły Biznesu.

Sobrero, A.A., \& Miglietta, A. (2006). Introduzione alla linguistica italiana. Roma/Bari: Laterza.

Widłak, S. (2012). Regionalismi e dialettismi. La loro presenza nell'uso comune e nella letteratura polacca. In G. Marcato (Ed.), Scrittura, dialetto e oralità (pp. 72-84). Padova: Coop. Libraria Editrice Università di Padova.

Wilkoń, A. (2000). Typologia odmian językowych współczesnej polszczyzny. Katowice: Wydawnictwo Uniwersytetu Śląskiego.

\section{Corpus}

Aukenthaler, H., Rienzner, W., Heidegger, A., Gerstgrasser, L., \& Raffl, U. (2010). Conoscere la selvaggina. Ambiente - biologia - caccia. Bolano: Athesia.

Becchi, A. (2009, July). Apertura alla lepre con i segugi. Caccia apertura 2009, 2(2009),74-82.

Biały, K., \& Jóźwiak, Z. (1994). Słownik podstawowych terminów łowieckich i ekologicznych. Warszawa: Łowiec polski. 
Calibro, M. (2012). Caccia alla lepre: un mondo di emozioni. Retrieved from http://bighunter.it/Home/Editoriale/Editoriale/tabid/204/newsid730/11771/Default.aspx.

Camusso, R. (1997, August). La lepre è intelligente? Sentieri di caccia, 8(III), 20-29.

Casanova, P., Capacciolo, A., \& Cellini, L. (1993). Appunti di zoologia venatoria e gestione della selvaggina. Firenze: Polistampa.

Farini, P., \& Ascari, A. (1941). Dizionario della lingua italiana di caccia. Milano: Garzanti.

Hoppe, S. (1966). Słownik języka łowieckiego. Warszawa: PWN.

Hoppe, S. (1981). Stownik języka łowieckiego. Warszawa: Państwowe Wydawnictwo Rolnicze i Leśne.

Klimowicz, R. (2017). Zając szarak. Retrieved from http://www.rys.elk. pl/2018/07/30/zajac-szarak.

Konieczny, K. (2019, February 22). Właśnie rodzą się małe zające - nie dotykaj, nie zabieraj!! [Blog post]. Retrieved from http://dbajobociany. $p l / ? p=14383$.

Kozłowski, W. (1822). Pierwsze początki terminologii łowieckiej. Warszawa: Drukarnia N. Glücksberga, Księgarza i Typografa Królewskiego Uniwersytetu.

Krzemień, M.P. (1984). 1000 słów o łowiectwie. Warszawa: Wydawnictwo Ministra Obrony Narodowej.

Marzola, S. (2013). Alla lepre con i fermatori. Retrieved from www.cacciando. $\mathrm{com} /$ caccia/tipi-di-caccia/stanziale-e-vagante/item/1240-alla-leprecon-i-fermatori.htlm.

Meloni, P. (2011). La lepre: tecniche di caccia alla lepre. Retrieved from www. cacciapassione.com/la-lepre-tecniche-di-caccia-alla-lepre.

Pasławski, T. (1973). Łowiectwo dla leśników i myśliwych. Warszawa: Państwowe Wydawnictwo Rolnicze i Leśne.

Pasławski, T. (2002). Metody polowań - myśliwskie rady i sposoby. Warszawa: Świat.

Poradnik łowiecki (2019). Zając szarak. Retrieved from https://poradniklowiecki.pl/component/content/article/210-zwierzyna/1080-zajac-szarak. html?highlight $=$ WyJ6d2llcnp5bmEiLCJzemFyYWsiXQ==.

Ugolini, L. (1961). Il Dizionario del cacciatore italiano. Milano: Bietti.

Zennaro, G. (2014). Risultati produttivi e riproduttivi di un allevamento di lepri nella regione Veneto [Tesi di Laurea]. Padova: Università degli Studi di Padova. 
Żeromski, H.B. (1990). Słownik łowiecki polsko-niemiecki i niemiecko-polski. Warszawa: Wydawnictwo Naukowo-Techniczne.

Brać łowiecka - miesięcznik myśliwych i sympatyków łowiecka; numeri dagli anni: 2016-2018.

Łowiec Polski - miesięcznik PZE, numeri dagli anni: 2015-2018.

Riassunto: Il presente articolo espone sia i risultati ottenuti durante la raccolta dei lessemi relativi alla lepre sia la loro analisi comparativa. L'interesse principale è stato posto sull'analisi semantica dei nomi e degli aggettivi riguardanti le specie di lepre e i singoli animali, le abitudini di vita, il sesso, l'età e i tratti del carattere leprino. Tutti questi lessemi fanno parte del linguaggio speciale venatorio. Considerando la ricchezza lessicale del materiale e la sua varietà interna, il corpus è stato ricavato dai dizionari venatori polacchi e italiani del XIX e XX secolo, nonché dalle fonti più recenti e informali: forum e blog dedicati alla caccia. I risultati avvalorano l'ipotesi dell'esistenza di una terminologia molto ramificata ed estesa nelle lingue analizzate. Tuttavia, sono state riscontrate alcune differenze nella struttura dei campi lessicali, come pure discrepanze nel numero delle espressioni relative alla lepre in polacco e in italiano.

Parole chiave: nomi, aggettivi qualificativi, lepre, lingua venatoria, equivalenza semantica 